\title{
Supplementary Information of Distinguishing the Structure of High-Pressure Hydrogen with Dielectric Constants
}

Da Chen, Ting Ting Cui,* Wang Gao,* and Qing Jiang

Key Laboratory of Automobile Materials, Ministry of Education, and School of Materials Science and Engineering, Jilin University, Changchun 130022, China

*Email: cuiting0228@sina.com.

*Email:wgao@jlu.edu.cn.

\section{Computational details}

The static calculations were performed using two different functionals in the castep plane-wave code: ${ }^{1}$ the generalized gradient approximation (GGA) functional of Perdew-Burke-Ernzerhof $(\mathrm{PBE})^{2}$ and the hybrid functional of Heyd-ScuseriaErnzerhof (HSE). ${ }^{3}$ For PBE calculations, we chose the ultrasoft pseudopotential with the plane-wave energy cutoff of $1200 \mathrm{eV}$ and the Monkhorst-Pack Brillouin ${ }^{4}$ zone sampling grid of spacing $2 \pi \times 0.025 \AA^{-1}$. The convergence thresholds of the force on atoms and the max stress were $9 \times 10^{-4} \mathrm{eV} / \AA$ and $0.01 \mathrm{GPa}$, respectively, to ensure the energy converges better than $0.1 \mathrm{meV}$ per proton. For HSE calculations, we used the norm-conserving pseudopotential with the plane-wave energy cutoff of $600 \mathrm{eV}$ and the Monkhorst-Pack Brillouin zone sampling grid of spacing $2 \pi \times 0.06 \AA^{-1}$. The force and the max stress on atoms were less than $0.01 \mathrm{eV} / \AA$ and $0.01 \mathrm{GPa}$, respectively. Moreover, 
HSE calculations were performed in cells containing 12 atoms for Cmca-12, 16 atoms for $P 6_{3} / m m c$ and $C m c 2_{1}, 24$ atoms for $P 2_{1} / c-24$ and $C 2 / c-24,32$ atoms for $P 6_{3} / m, P c a 2_{1}$ and $P 6_{1} 22,{ }^{5-7}$ after the careful test of the size effect of super-cell. In particular, we calculated with super-cells containing 96 atoms for $P 2_{1} / c-24,128$ atoms for $P 6_{3} / m$ and $\mathrm{Pca}_{1}$ (phase II), 96 atoms for $C 2 / c-24$ and 128 atoms for $P 6{ }_{1} 22$ (phase III) to ensure the size effect no more than $1 \mathrm{meV} /$ atom. The vdW interactions were calculated by pairwise Tkatchenko and Scheffer (TS) ${ }^{8}$ and many-body dispersion (MBD) ${ }^{9}$ methods, respectively.

The phonon calculations were carried out with PBE functional and the finitedisplacement method. We performed geometry optimization and quasiharmonic phonon calculations using on the fly pseudopotential with the plane-wave energy cutoff of $1200 \mathrm{eV}$ and the Monkhorst-Pack Brillouin zone sampling grid of spacing $2 \pi \times 0.05$ $\AA^{-1}$. The convergence thresholds of the force on atoms and the max stress are $0.01 \mathrm{eV} / \AA$ and 0.01 GPa, respectively. To ensure the harmonic free energies converge to less than $1 \mathrm{meV}$ per proton, we calculated in super-cells containing 256 atoms for $P 6_{3} / \mathrm{mmc}$ and $C m c 2_{1}, 128$ atoms for $P 6_{3} / m, 64$ atoms for $P c a 2_{1}, 192$ atoms for $P 2_{1} / c-24$ and $C 2 / c-24$, 96 atoms for Cmca-12, and 288 atoms for $P 6_{1} 22$.

The dielectric constant calculations were performed using two different methods, respectively. One is implemented using density functional perturbation theory $(\mathrm{DFPT})^{10}$ with GGA functional of PBE augmented with the MBD method as calculated in the VASP package. ${ }^{11}$ The local field effect is considered to include microscopic changes of the exchange-correlation potential. To ensure the convergence of dielectric 
constant results, the Brillouin zone sampling is used with the Monkhorst-Pack method with $16 \times 16 \times 16$ grid and the plane-wave energy cutoff is set to $1200 \mathrm{eV}$ with projector-augmented wave (PAW) pseudopotential ${ }^{12}$ which achieve the all-electron pseudopotential accuracy with lower plane-wave cutoff. The other is implemented based on the Clausius-Mossotti (CM) equation ${ }^{13}$ using GGA functional of PBE augmented with vdW ${ }^{14,15}$ method as calculated in the CASTEP code. The plane-wave energy cutoff of $1200 \mathrm{eV}$ and the Monkhorst-Pack Brillouin zone sampling grid of spacing $2 \pi \times 0.025 \AA^{-1}$ is used after the convergence test.

For the molecular dynamics (MD) simulations, we used NVT ensembles with the plane-wave energy cutoff of $600 \mathrm{eV}$ and the Monkhorst-Pack Brillouin zone sampling grid of spacing $2 \pi \times 0.05 \AA^{-1}$. The time step is 0.5 fs. The simulations were performed in super-cells containing 144 atoms for both the $C 2 / C$-24 and $P 6_{1} 22$ with the primitive cell at $240 \mathrm{GPa}$. The temperature was chosen at $250 \mathrm{~K}$, about $50 \mathrm{~K}$ lower than the transition temperature between phases III and IV. After an equilibrium period of about 0.3 ps, we sampled four static data points for each structure. 


\section{Nonlocal electron exchange contributions}

(a)

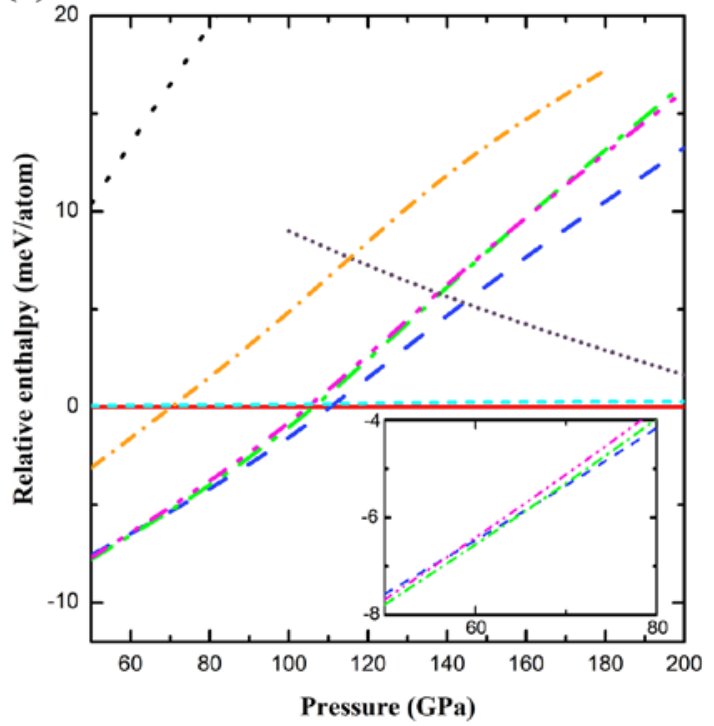

(b)

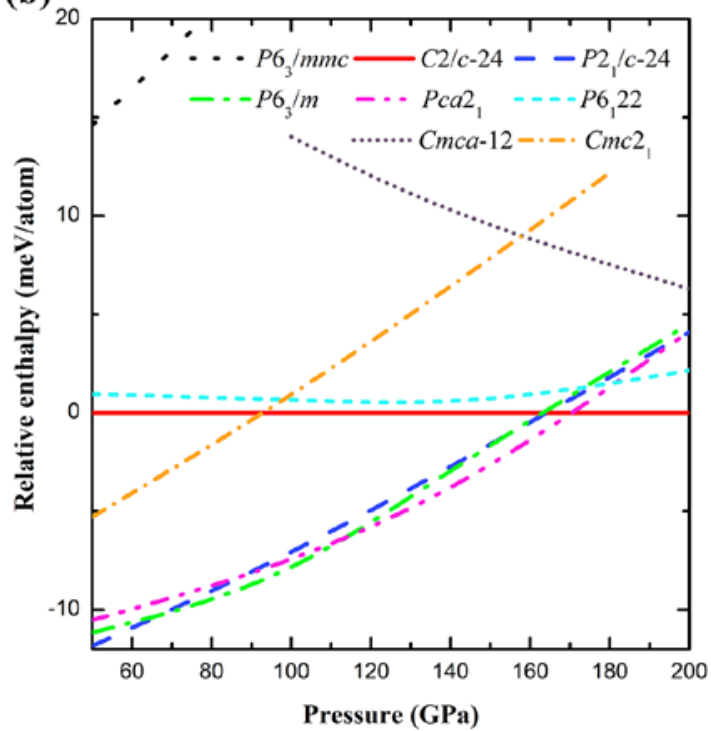

Figure S1. Relative enthalpy per atom as a function of pressure by different functionals:

(a) PBE and (b) HSE. The static lattice enthalpies of high-pressure hydrogen are calculated relative to the $C 2 / c-24$ structure. The inset shows the enlarged view for the low pressure in (a).

We investigate the effects of nonlocal electron exchange on the phase diagram by comparing the results of PBE and HSE functionals, showing the results in Figure S1. The nonlocal electron exchange greatly increases the transition pressure but can hardly distinguish the candidate structures of phase II. According to our PBE calculations shown in Figure S1a, the most stable phases are $P 6_{3} / m$ ( $\left.<65 \mathrm{GPa}\right), P 2_{1} / c-24(65-111$ $\mathrm{GPa}$ ) and $C 2 / c-24(>111 \mathrm{GPa})$, respectively, which are consistent with previous results of Pickard and Needs. ${ }^{5,16}$ In contrast, as shown in Figure S1b, HSE calculations predict the phase transition in the order of $P 2_{1} / c-24(<70 \mathrm{GPa}) \rightarrow P 6_{3} / m(70-114 \mathrm{GPa}) \rightarrow P c a 2_{1}$ (114-169 GPa) $\rightarrow$ C2/c-24 (>169 GPa). The nonlocal electron exchange has little effect 
on the energy differences (less than $2 \mathrm{meV}$ per atom at $150 \mathrm{GPa}$ ) between the candidate structures of phase II $\left(P 2_{1} / c-24, P 6_{3} / m\right.$ and $\left.P c a 2_{1}\right)$, which manifest their similar degree of electron delocalization. On the contrary, the energy difference between $P 6_{3} / m$ and C2/c-24 (the candidate structures of phases II and III, respectively) changes significantly (about $9 \mathrm{meV}$ per atom at $150 \mathrm{GPa}$ ), due to their large difference in the degree of electron delocalization which could be reflected by their band gaps difference (about $0.4 \mathrm{eV}$ at $150 \mathrm{GPa}$ calculated using BLYP functional). ${ }^{17}$ The nonlocal electron exchange greatly increases the transition pressure between phases II and III by about $58 \mathrm{GPa}$ and gives a result closer to the experimental ones. ${ }^{18-21}$ As the pressure increases, the nonlocal electron exchange also immensely increases the transition pressure of $C 2 / c-24 \rightarrow C m c a-12$, associated with their large difference of band gaps (about $0.7 \mathrm{eV}$ at 200 GPa from BLYP calculation results). ${ }^{17}$ Furthermore, as another candidate for phase III, $P 6_{1} 22$ becomes even more unstable relative to $C 2 / c-24$ with the inclusion of nonlocal electron exchange, especially at high pressures (above $160 \mathrm{GPa}$ ). 


\section{Van der Waals contributions}
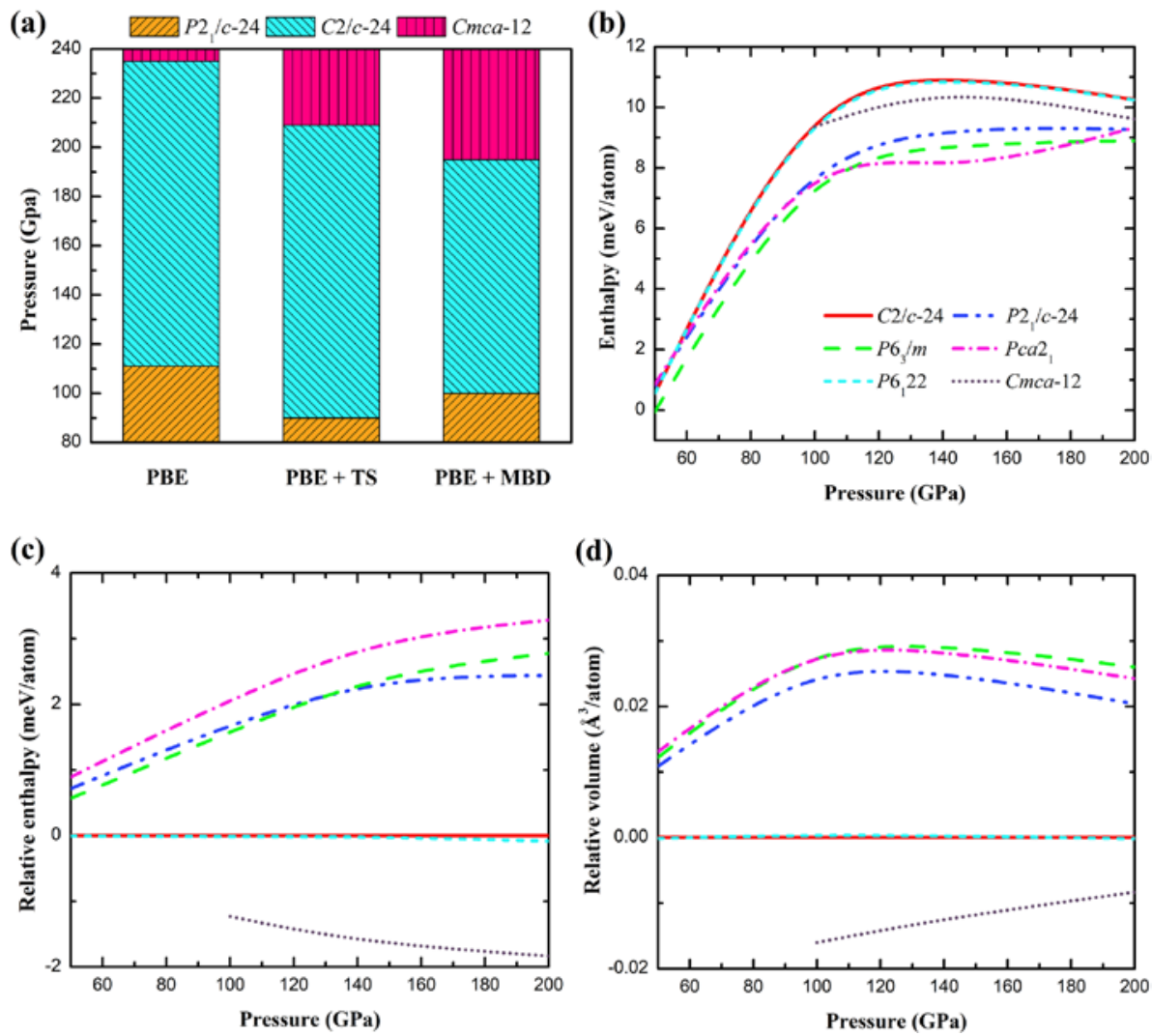

Figure S2. (a) Static phase diagrams calculated by PBE, PBE augmented with TS (PBE+TS) and PBE augmented with MBD (PBE+MBD) methods, respectively, (b) the energy difference between MBD and TS results $\left(E_{\mathrm{MBD}}-\mathrm{TS}\right)$ of candidate structures of phases II $\left(P 2_{1} / c-24, P 6_{3} / m\right.$ and $\left.P c a 2_{1}\right)$ and III (C2/c-24, P6122 and Cmca-12), respectively, (c) and (d) the relative MBD energy difference and relative volume of phase II and III as a function of pressure, respectively.

It is well known that vdW interactions are essential for the formation, stability of molecular structures, ${ }^{8}, 9,22$ however, the general DFT couldn't describe vdW interactions properly. We use TS and MBD methods to revise the phase diagram, respectively. It is shown that both $\mathrm{PBE}+\mathrm{TS}$ and $\mathrm{PBE}+\mathrm{MBD}$ methods predict the same 
phase order of $P 2{ }_{1} / c-24 \rightarrow C 2 / c-24 \rightarrow C m c a-12$ as PBE method but with lower transition pressure. As shown in Figure S2a, the TS correction reduces the transition pressure of $P 21 / c-24 \rightarrow C 2 / c$-24 by $21 \mathrm{GPa}$ (from $111 \mathrm{GPa}$ to $90 \mathrm{GPa}$ ), which is bigger than the reduction of $11 \mathrm{GPa}$ by MBD correction (from $111 \mathrm{GPa}$ to $100 \mathrm{GPa}$ ). Conversely, for the transition pressure of $C 2 / c-24 \rightarrow C m c a-12$, the TS correction reduces the transition pressure by 26 GPa (from 235 GPa to 209 GPa), obviously smaller than the reduction of $40 \mathrm{GPa}$ by MBD correction (from $235 \mathrm{GPa}$ to $195 \mathrm{GPa}$ ). It is known that TS method relies on a summation over a pairwise interatomic $C_{6} / R^{6}$ term damped at short distances. ${ }^{8} C_{6}$ is the integral sum of atomic polarizability and $R$ is the distance between pairs of atoms. On the basis of TS method, MBD method includes two additive parts: screening effect in the medium range and high-order correlation in the long range. ${ }^{9,} 23$ We can study the disparity between the effect of MBD and TS methods on the phase diagram through Figure S2b. At low pressure (<60 GPa), the energy differences between MBD and TS results ( $\left.\Delta E_{\mathrm{MBD}}-\mathrm{TS}\right)$ of various structures tend to be zero, while as the pressure increases, $\Delta E_{\mathrm{MBD}}$ - Ts values increase rapidly, corresponding to the minor many-body effects of vdW forces at low pressure and the major many-body effects of vdW forces at high pressure. These results reflect the fact that the electron overlaps between hydrogen molecules of the considered hydrogen phases are negligible at low pressure but significant at high pressure. Notably, $\Delta E_{\mathrm{MBD}-}$ Ts differences between the candidate structures of phase II $\left(P 2_{1} / c-24, P 6_{3} / m\right.$ and $\left.P c a 2_{1}\right)$ and phase III (C2/c-24, $P 6_{12} 22$ and $\left.C m c a-12\right)$ are large enough (could reach a maximum of $3 \mathrm{meV}$ per atom) to be comparable with the differences between their PBE (or HSE) 
energies above 100 GPa. Therefore, we choose the MBD method to study the vdW effect on the phase diagram of high-pressure hydrogen.

The role of vdW forces during phase transition becomes more and more significant with pressure and is structurally dependent. As illustrated in Figure S2c, $P 2{ }_{1} / c$-24, $P 6_{3} / m$ and $P c a 2_{1}$ structures have comparable MBD energies, which is ascribed to their similar interatomic distances and configurations. The interatomic distances can be reflected through volumes and Figure S2d shows that these three structures have similar volumes. The $P 6_{3} / m, P 2_{1} / c-24$ and $P c a 2_{1}$ are hcp, monoclinic and orthorhombic structures, respectively, while their molecular centers are still on or near the hcp lattice. Small differences in molecular arrangements and interatomic distances yield little effect on their phase stability with the inclusion of MBD interactions. As pressure increases, $P 21 / c$-24 translates to a compact structure of $C 2 / c$-24 with the molecular arrangements deviated from hcp lattice. Smaller interatomic distances of $C 2 / c-24$ than that of $P 2{ }_{1} / c$ 24 structure result in stronger MBD interactions of $C 2 / c-24$, shown in Figure S2c. Moreover, the MBD energy difference between $P 22_{1} / c-24$ and $C 2 / c-24$ structures increases with pressure and rises to $1.7 \mathrm{meV} /$ atom at $100 \mathrm{GPa}$ which leads to the reduction in their transition pressure by $11 \mathrm{GPa}$. As another competitive candidate structure of phase III, $P 6_{12} 22$ has almost the same equation of state and the same MBD energy with $C 2 / c$-24 due to their similar structures. ${ }^{7}$ As the pressure increases further, C2/c-24 transforms to $\mathrm{Cmca-12}$ structure with smaller interatomic distances, resulting in stronger MBD interactions in $C m c a-12$ than $C 2 / c$-24. Interestingly, the MBD energy difference between $C 2 / c$-24 and Cmca-12 structures also increases with pressure and 
reaches $1.8 \mathrm{meV} /$ atom at $195 \mathrm{GPa}$, decreasing the transition pressure by as high as 40 GPa. Hence, it is imperative to study the phase diagram of high-pressure hydrogen considering the effect of van der Waals forces.

\section{Zero-point energy contributions}

(a)

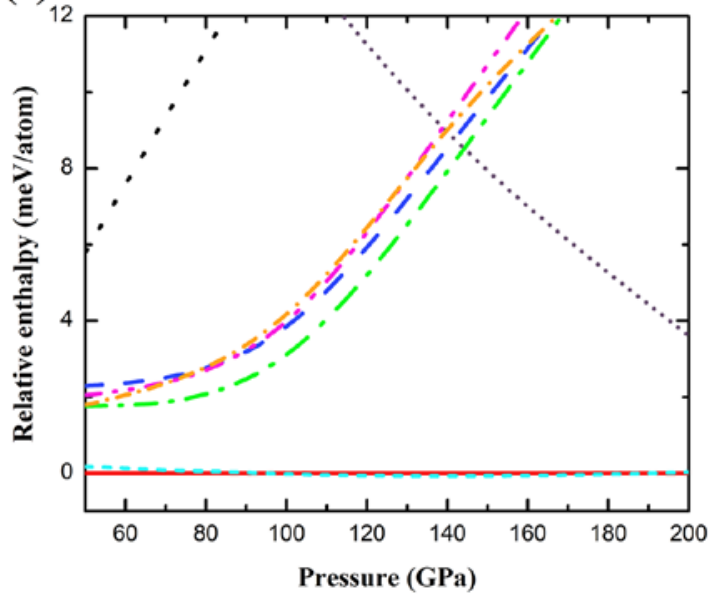

(b)

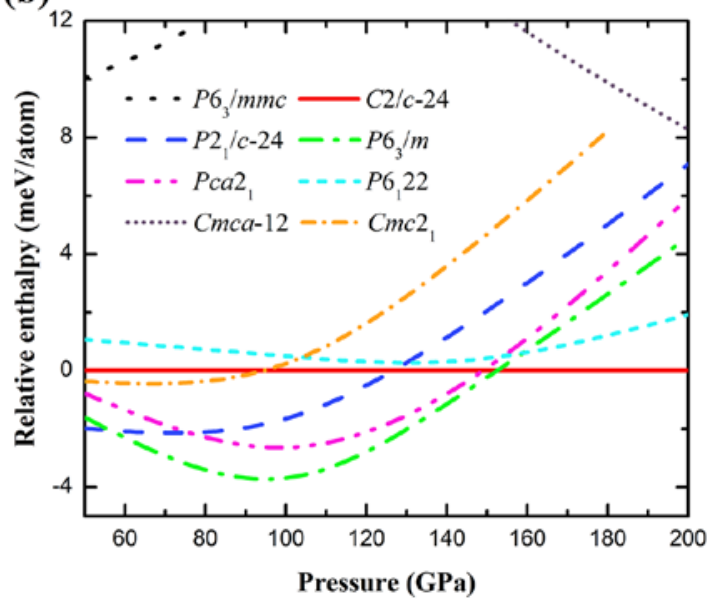

Figure S3. Enthalpy per atom including ZPE of high-pressure hydrogen as a function of pressure, relative to the $C 2 / c-24$ structure. (a) PBE+ZPE, (b) HSE+ZPE.

Based on the phase diagram by static calculations, we calculated the zero-point energy within harmonic approximation. It is shown that ZPE has different effects on the transition pressure for various structures and even changes the phase order (see Figures S1 and S3). ZPE dramatically reduces the transition pressure of phases II and III, and PBE+ZPE results predict no stable pressure range for the candidate structures of phase II, which accords with the previous dynamical results by Pickard and Needs. ${ }^{5}$ In contrast, HSE+ZPE results show the existence of phase II, which indicates the nonlocal electron exchange is significant not only for the magnitude of transition 
pressure but also for phase stability. The transition pressure of phase II-III decreases from $164 \mathrm{GPa}$ to $154 \mathrm{GPa}$ with the inclusion of ZPE and agrees well with the experimental result of $150 \mathrm{GPa} .{ }^{18-21}$ On the other hand, ZPE increases the transition pressure of $C 2 / c-24 \rightarrow C m c a-12$ obviously and the resulting transition pressure by HSE+ZPE becomes close to previous DMC results. ${ }^{24}$ Especially, for structures except Cmca-12, ZPE makes the HSE curves of various structures get closer and bring difficulty to phase discrimination.

\section{Gibbs free energy phase diagram}

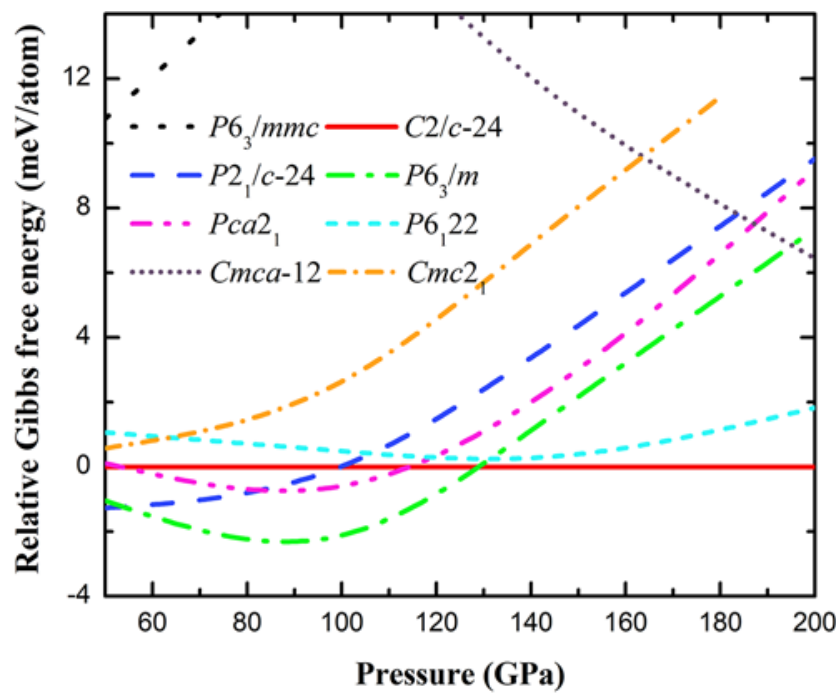

Figure S4. Relative Gibbs free energy of high-pressure hydrogen calculated by HSE functional together with ZPE and MBD interactions. 


\section{Dependence of the x-ray diffraction pattern on functional}
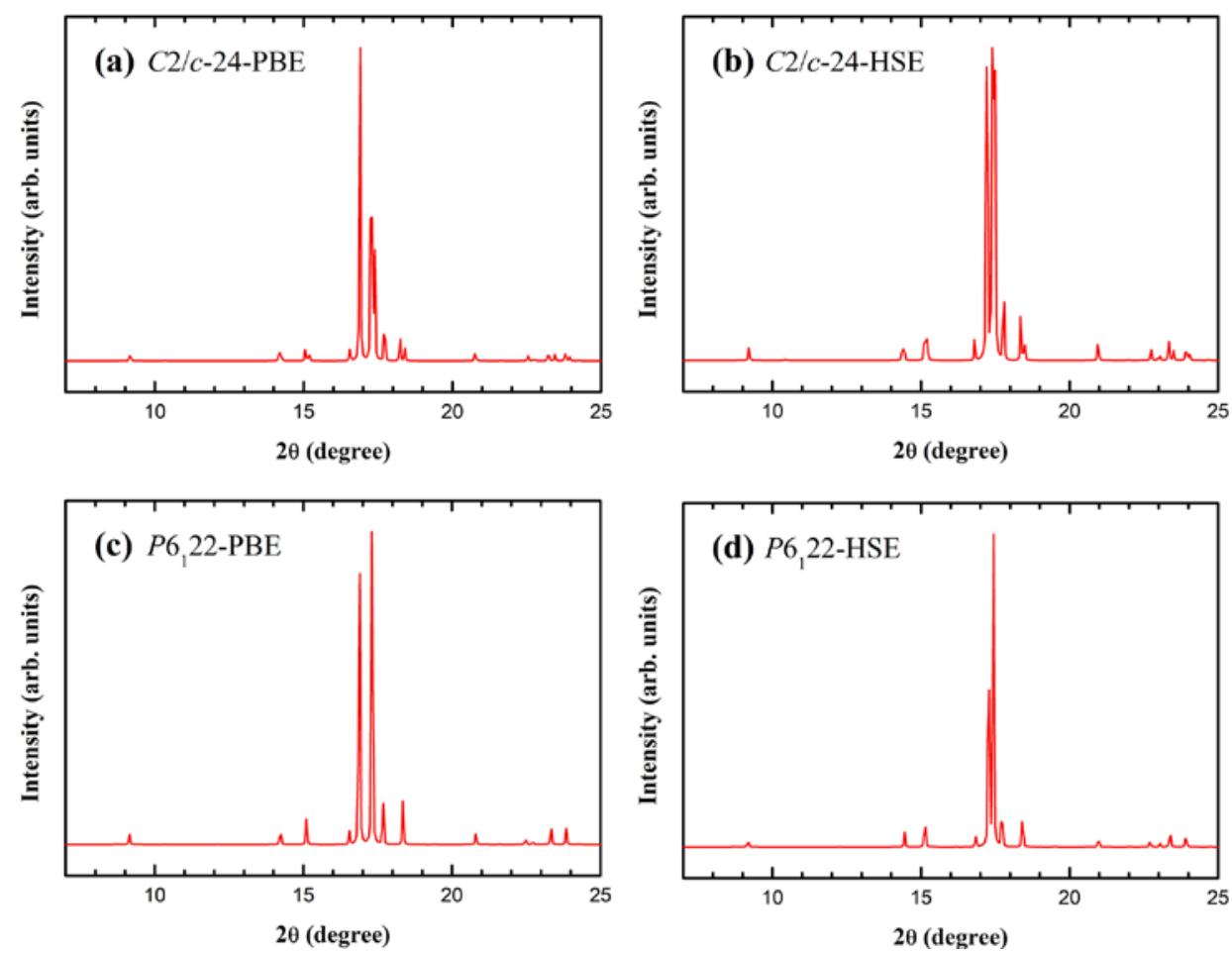

Figure S5. X-ray powder-diffraction patterns of $C 2 / c-24$ and $P 6{ }_{1} 22$ structures by different functionals at $140 \mathrm{GPa}$ and wavelength $\lambda=0.4122 \AA$.

We investigate the XRD patterns of the $C 2 / c$-24 and $P 6_{1} 22$ structures use PBE and HSE functionals, respectively. Our calculated XRD data indicate that the angle, the intensity, and even the number of diffraction peaks depend strongly on the functionals. In general, the diffraction peak angles increase with pressure due to the decrease of surface spacing according to the Bragg equation $2 d \sin \theta=n \lambda$. As shown in Figure S5, the double-diffraction peak of $C 2 / c-24$ structure optimized by PBE functional $(C 2 / c$ 24-PBE) around $15.1^{\circ}$ at $140 \mathrm{GPa}$ (corresponding to the double diffraction-peak about $15.5^{\circ}$ at $174 \mathrm{GPa}$ of previous BLYP calculations ${ }^{7}$ ) transform into a single-diffraction peak with the inclusion of nonlocal electronic exchange (C2/c-24-HSE), which is 
almost identical with the diffraction peak of $P 6_{1} 22$ (P6 $\left.22-H S E\right)$. Furthermore, the nonlocal electronic exchange significantly changes the intensity and increases the angle of the diffraction peak above $16^{\circ}$ which is mainly related to the overestimation of the surface spacing by PBE functional. For instance, when the functional changes from PBE to HSE, the surface spacing of 101 crystal face for $C 2 / c$-24 structure decreases from $1.403 \AA$ to $1.377 \AA$ A. As illustrated in Figures S5a and S5b, the diffraction peak angle of $C 2 / c$-24 at $17.2^{\circ}$ increases to $17.4^{\circ}$ and the intensity doubles with the inclusion of nonlocal electronic exchange. Similarly, the diffraction peak of $P 6_{1} 22$ also shifts to the right, but its peak intensity at $17.2^{\circ}$ hardly changes only with a significant decrease in peak strength of the other diffraction peak at $16.9^{\circ}$. Therefore, the XRD patterns of $C 2 / c-24$ and $P 6{ }_{1} 22$ have different dependence on functionals. Moreover, there are many other factors on XRD patterns that need to be carefully tested, such as vdW correction (always reduce the volume and surface spacing), quantum nuclear vibrational motion (due to the low mass of hydrogen), and temperature (the experimental results are at 100 $\mathrm{K})$. Experimentally, it is also challenging to obtain reliable XRD data of high-pressure hydrogen due to its weak X-ray scattering properties. Hence, the comparison between experimental and theoretical XRD patterns would be difficult, especially in distinguishing the similar structures such as $P 6_{12} 22$ and $C 2 / c-24$. 


\section{Diagonalization of the dielectric matrix}
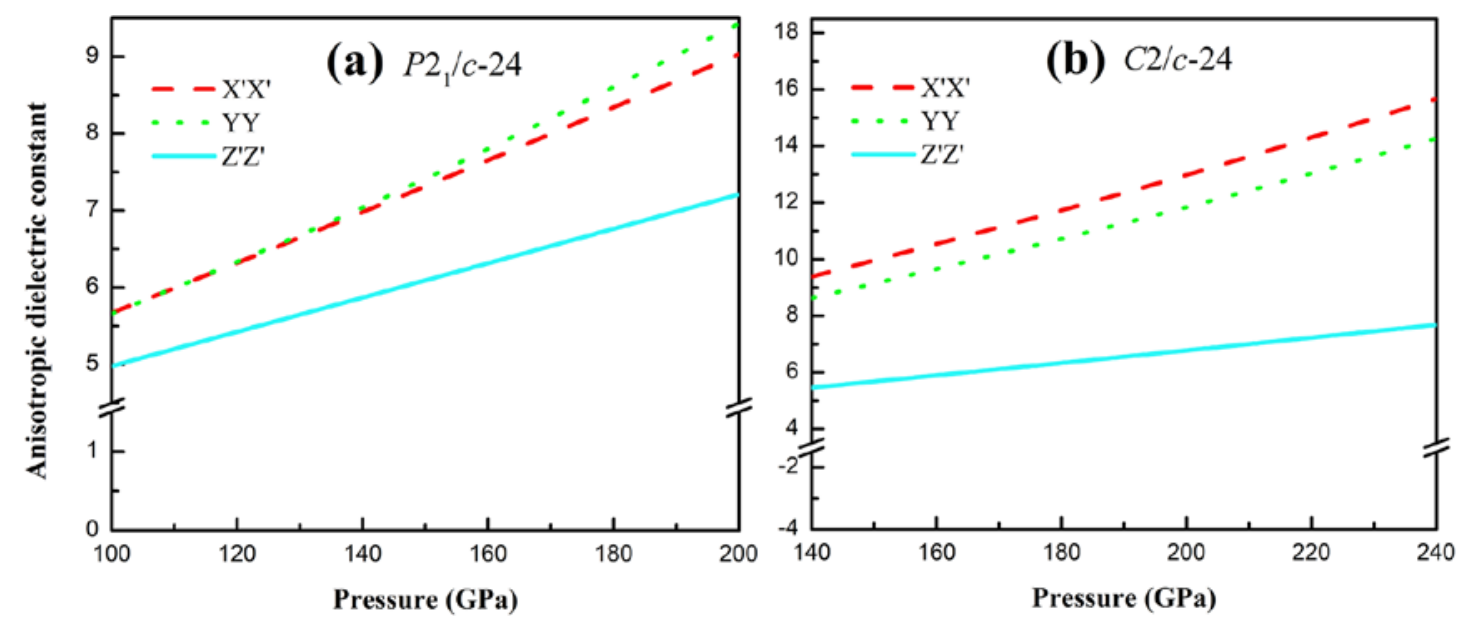

Figure S6. Anisotropic dielectric constant of $P 2{ }_{1} / c-24$ and $C 2 / c-24$ structures after diagonalization of the dielectric matrix calculated by PBE+MBD method based on density functional perturbation theory.

The dielectric matrix depends on the selection of the lattice axis. For the structure with the direction of spontaneous polarization deviating from the crystal axis, the diagonalizable dielectric matrix can be obtained if we choose the direction of spontaneous polarization as the new axis. Such as for the monoclinic structures of $P 2{ }_{1} / c$-24 and $C 2 / c-24$, when an electric field is applied along the $\mathrm{X}$ or $\mathrm{Z}$ axis, the spontaneous polarization deviates from the $\mathrm{X}$ or $\mathrm{Z}$ axis. Therefore, we can redefine their corresponding spontaneous-polarization directions as the new $\mathrm{X}^{\prime}$ and $\mathrm{Z}^{\prime}$ axes to obtain the diagonalizable permittivity matrix containing three dielectric constant tensors: $\varepsilon_{X} x^{\prime}$, $\varepsilon_{\mathrm{YY}}$ and $\varepsilon_{\mathrm{Z} Z}$. The diagonalizable dielectric matrix can be obtained by solving the eigenvalue of the matrix, and the corresponding new coordinate axes can be transformed by solving the eigenvector of the dielectric matrix. For the mathematical 
details, please refer to the diagonalization method of the matrix. As shown in Figure S6a, the diagonalizable permittivity tensors of $P 2{ }_{1} / c-24$ change slightly compared with that before diagonalization due to its small off-diagonal dielectric constant tensor $\varepsilon_{\mathrm{Xz}}$. However, from the perspective of structural detection, the diagonalizable dielectric tensor of $P 2_{1} / c-24$ is similar to that of $P 6_{3} / m$ below $140 \mathrm{GPa}$ and that of $P c a 2_{1}$ at higher pressures (see Figures 3a and c). Therefore, the measurement of the diagonalizable dielectric tensor is unfavorable to the structural detection of phase II, instead, the measurement of dielectric tensor $\varepsilon_{\mathrm{xz}}$ is recommended. In contrast, after diagonalization, the diagonal dielectric tensors of $C 2 / c$-24 change obviously due to its large negative off-diagonal dielectric tensor $\varepsilon_{\mathrm{XZ}}$. The dielectric constant $\varepsilon_{\mathrm{YY}}$ is no longer the largest value but less than the dielectric constant $\varepsilon_{X}$ '. Moreover, all the diagonal dielectric constants are positive, eliminating the doubt about our calculation results for the dielectric constant of $C 2 / c$-24 (the existence of negative dielectric constant $\varepsilon_{\mathrm{Xz}}$ ). Importantly, the three different diagonal dielectric tensors of $C 2 / c$-24 remain distinct obviously from the two independent diagonal dielectric tensors of $P 6_{1} 22$. That is to say, $C 2 / c-24$ exhibits different dielectric properties compared to $P 6_{1} 22$, independent on the choice of principal axis. Therefore, the measurement of anisotropic dielectric constant provides a potential means to probe the structure of high-pressure hydrogen. 


\section{Thermal effects on the dielectric tensors}
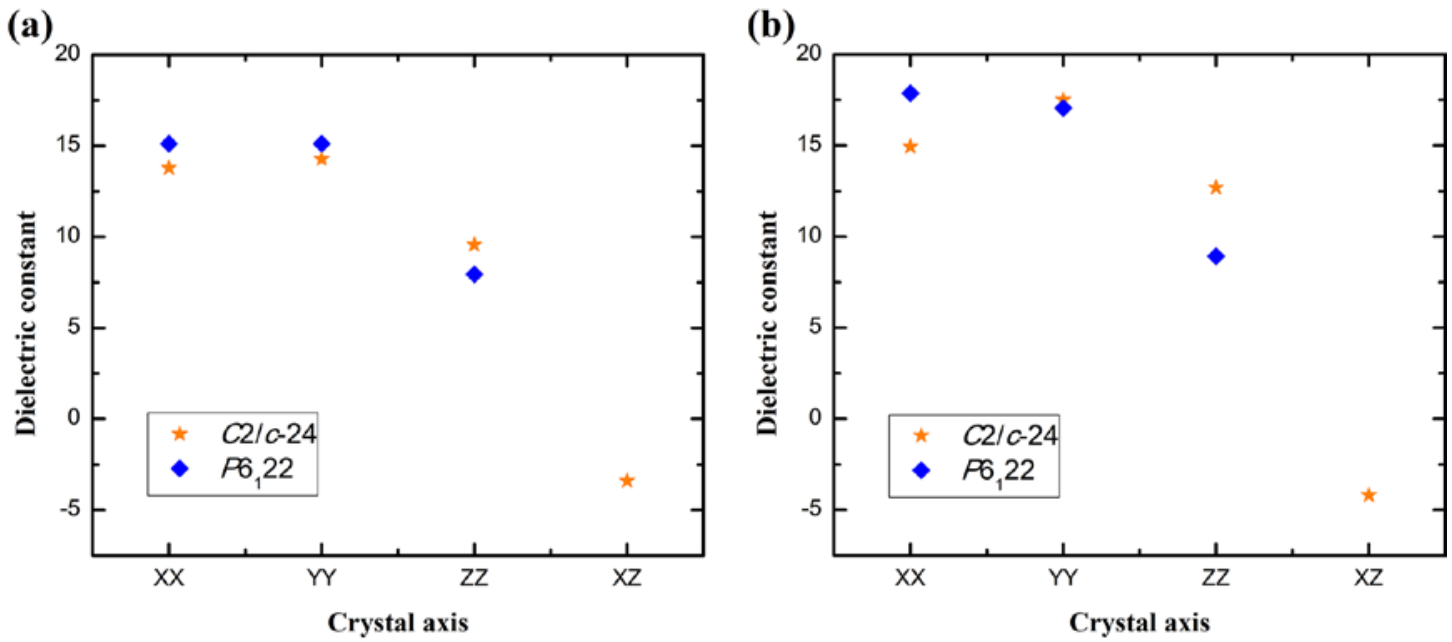

Figure S7. Dielectric tensors of $C 2 / c-24$ and $P 6_{1} 22$ (a) without the thermal effects and

(b) with the thermal effects. 


\section{REFERENCES}

1. Clark, S. J.; Segall, M. D.; Pickard, C. J.; Hasnip, P. J.; Probert, M. I. J.; Refson, K.; Payne, M. C., First principles methods using CASTEP. Z. Kristallogr. 2005, 220, 567. 2. Perdew, J. P.; Burke, K.; Ernzerhof, M., Generalized Gradient Approximation Made Simple. Phys. Rev. Lett. 1996, 77, 3865.

3. Krukau, A. V.; Vydrov, O. A.; Izmaylov, A. F.; Scuseria, G. E., Influence of the exchange screening parameter on the performance of screened hybrid functionals. $J$. Chem. Phys. 2006, 125, 224106.

4. Monkhorst, H. J.; Pack, J. D., Special points for Brillouin-zone integrations. Phys. Rev. B 1976, 13, 5188.

5. Pickard, C. J.; Needs, R. J., Structure of phase III of solid hydrogen. Nat. Phys. 2007, 3, 473.

6. Geneste, G.; Torrent, M.; Bottin, F.; Loubeyre, P., Strong isotope effect in phase II of dense solid hydrogen and deuterium. Phys. Rev. Lett. 2012, 109, 155303.

7. Monserrat, B.; Needs, R. J.; Gregoryanz, E.; Pickard, C. J., Hexagonal structure of phase III of solid hydrogen. Phys. Rev. B 2016, 94, 134101.

8. Tkatchenko, A.; Scheffler, M., Accurate Molecular Van Der Waals Interactions from Ground-State Electron Density and Free-Atom Reference Data. Phys. Rev. Lett. 2009, 102, 073005.

9. Ambrosetti, A.; Reilly, A. M.; DiStasio, R. A., Jr.; Tkatchenko, A., Long-range correlation energy calculated from coupled atomic response functions. J. Chem. Phys. 2014, 140, 18A508. 
10. Gajdoš, M.; Hummer, K.; Kresse, G.; Furthmüller, J.; Bechstedt, F., Linear optical properties in the projector-augmented wave methodology. Phys. Rev. B 2006, 73, 045112.

11. Kresse, G.; Furthmüller, J., Efficiency of ab-initio total energy calculations for metals and semiconductors using a plane-wave basis set. Comput. Mater. Sci. 1996, 6, 15.

12. Blöchl, P. E., Projector augmented-wave method. Phys. Rev. B 1994, 50, 17953.

13. Tessman, J. R.; Kahn, A.; Shockley, W., Electronic polarizabilities of ions in crystals. Phys. Rev. 1953, 92, 890.

14. Zhang, G.-X.; Tkatchenko, A.; Paier, J.; Appel, H.; Scheffler, M., Van der Waals interactions in ionic and semiconductor solids. Phys. Rev. Lett. 2011, 107, 245501.

15. Tkatchenko, A.; Distasio, R. A.; Car, R.; Scheffler, M., Accurate and Efficient Method for Many-Body van der Waals Interactions. Phys. Rev. Lett. 2012, 108, 236402. 16. Pickard, C. J.; Needs, R. J., Structures at high pressure from random searching. Phys. Status Solidi B 2009, 246, 536.

17. Azadi, S.; Foulkes, W. M. C., Fate of density functional theory in high-pressure solid hydrogen. Phys. Rev. B 2013, 88, 014115.

18. Mao, H. K.; Hemley, R. J., Ultrahigh-pressure transitions in solid hydrogen. Rev. Mod. Phys. 1994, 66, 671.

19. Dias, R. P.; Silvera, I. F., Observation of the Wigner-Huntington transition to metallic hydrogen. Science 2017, 357, 715.

20. Zha, C. S.; Liu, Z.; Hemley, R. J., Synchrotron infrared measurements of dense 
hydrogen to 360 GPa. Phys. Rev. Lett. 2012, 108, 146402.

21. Goncharov, A. F.; Hemley, R. J.; Mao, H., Vibron frequencies of solid H2 and D2 to $200 \mathrm{GPa}$ and implications for the P-T phase diagram. J. Chem. Phys. 2011, 134, 174501.

22. Cui, T. T.; Chen, D.; Li, J.; Gao, W.; Jiang, Q., Favoured Decomposition Paths of Hydrogen Sulfide at High Pressure. New J. Phys. 2019, 21, 033023.

23. Chattopadhyaya, M.; Hermann, J.; Poltavsky, I.; Tkatchenko, A., Tuning intermolecular interactions with nanostructured environments. Chem. Mater. 2017, 29, 2452.

24. Mcminis, J.; Clay, R. C.; Lee, D.; Morales, M. A., Molecular to Atomic Phase Transition in Hydrogen under High Pressure. Phys. Rev. Lett. 2015, 114, 105305. 